\title{
Design Rules to Optimize the Layout of Multilayer Circuit Packages at 100GHz
}

\author{
Anne D. Abeygunasekera and Charles Free \\ Advanced Technology Institute, University of Surrey \\ Guildford, Surrey, GU2 7XH, United Kingdom \\ a.abeygunasekera@surrey.ac.uk
}

\begin{abstract}
New data are presented on the effects of coupling between conductors in a highly integrated, multilayer circuit working at high millimeter wave frequencies. Design rules have been developed to summarize the results and provide guidance to the circuit designer on the minimum spacing between conductors in a multilayer package.
\end{abstract}

\section{Introduction}

The electrical behaviour of the interconnects is crucial in determining the performance of high speed digital, RF and microwave circuits. Low fabrication cost, and small size are two of the key requirements for hybrid microwave integrated circuits. Multilayer thick-film technology is a very attractive solution to address these needs. Circuit size may be greatly reduced by stacking the conductors, hence coupling between transmission lines on different levels of a multilayer structure become a critical design consideration. Whilst multilayer circuits offer many advantages for the microwave circuit designer, unwanted coupling due to high packing density may detract from the electrical performance of the multilayer package. Using electromagnetic simulation we have established criteria for the optimum spacing of conductors in multilayer ceramic packages at $100 \mathrm{GHz}$. The work was based around the use of photoimageable thick-film conductors and dielectrics; the dielectrics had a relative permittivity between 3.9 and 8 , and the conductors were silver. Three situations were considered in detail, firstly the effect of the crossover of conductors separated by dielectric, and secondly the effect of coupling between parallel conductors on different layers and finally the coupling between two right-angle conductor bends on different layers. Using the data obtained a program in Java was written to aid the design engineer to in finding the minimum spacing between conductors in a multilayer package.

\section{Cross-over Structure}

There are occasions where interconnection lines on different levels are required to cross, forming an four port network, as shown in Fig. 1. To avoid the crossing paths must be arranged in a manner that maintains adequate electrical isolation between the lines. The isolation can conveniently be represented in terms of the S-parameters of the four port network.

In order to make a practical investigation of the crossover effect a test circuit was fabricated on 10mil alumina, and the dielectric between the crossing lines formed from layers of printed thick-film, with $\mathrm{h}=40 \mu \mathrm{m}$, as shown in Fig.2. In the test structure, the upper and lower conductors each had a characteristic impedance of $50 \Omega$. Two different printed dielectrics were examined, having relative permittivities of 3.9 and 8 , respectively.

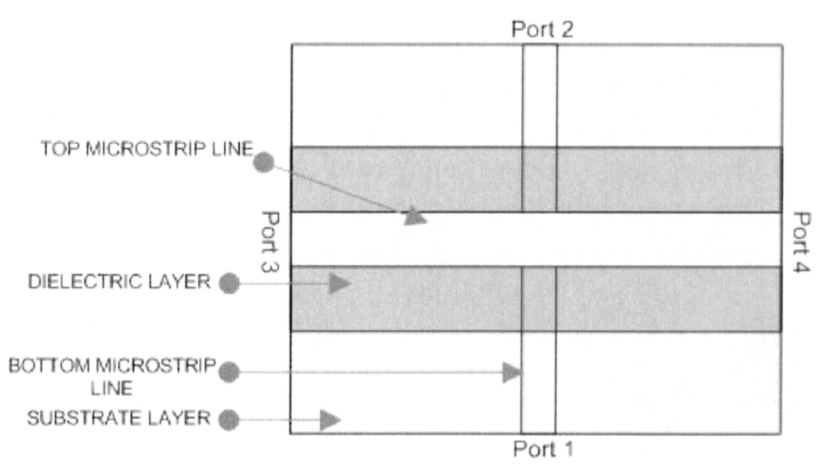

Fig. 1. Planar view of the cross-over structure

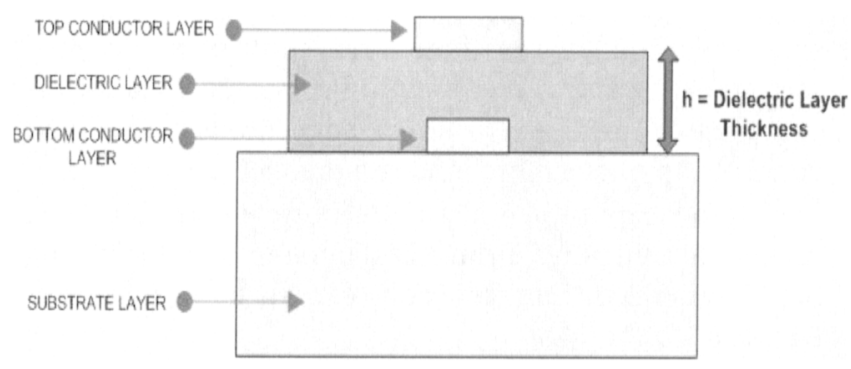

Fig. 2. Cross section view of the cross-over structure

The electrical performance of the multilayer crossover structure at $100 \mathrm{GHz}$ was studied using an EM simulator, namely Momentum ${ }^{\circledR}$ (AGILENT Advanced Design System). The simulated results are shown in Fig.3.

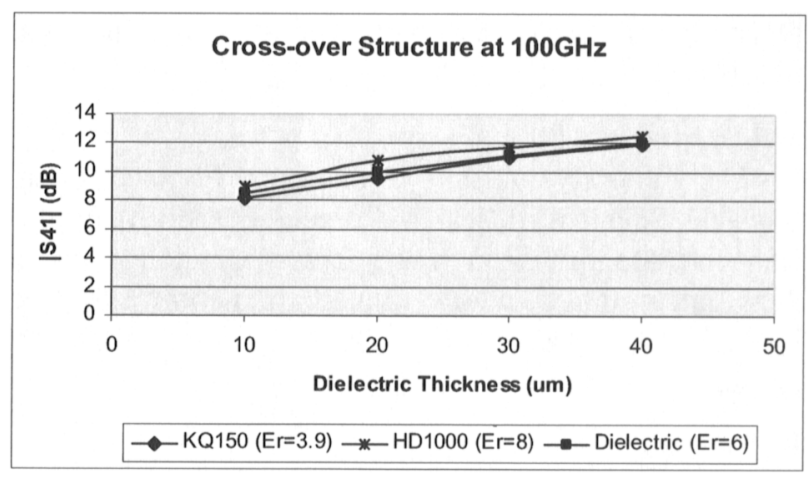

Fig. 3. Coupling of the cross-over structure 
It was found that the unwanted coupling between the crossed conductors varied nonlinearly with the thickness (h) of the separating dielectric layer. As expected, a decrease in coupling was observed when the dielectric thickness between the conductors was increased.

Using the data shown in Fig. 3, a curve fitting routine was used to determine relationships between the amount of coupling and the spacing, $h$, for different values of $\varepsilon_{\mathrm{r}}$.

For the conditions, where $|\mathrm{S} 11|>13 \mathrm{~dB},|\mathrm{~S} 12|<1 \mathrm{~dB}$, $|\mathrm{S} 14|>11 \mathrm{~dB},|\mathrm{~S} 33|>18 \mathrm{~dB}$ and $|\mathrm{S} 43|<1 \mathrm{~dB}$, and for $3.9 \leq$ $\varepsilon_{\mathrm{r}} \leq 8$, the minimum spacing required to achieve sufficient isolation between transmission lines, is given by:

$$
\begin{aligned}
& h=72.953-12.44 \varepsilon_{\mathrm{r}}-0.364 \varepsilon_{\mathrm{r}}^{2}+0.164 \varepsilon_{\mathrm{r}}^{3}+0.0228 \varepsilon_{\mathrm{r}}^{4}+ \\
& 0.0005 \varepsilon_{\mathrm{r}}^{5}-0.00028 \varepsilon_{\mathrm{r}}^{6}-0.00005 \varepsilon_{\mathrm{r}}^{7}-0.000003 \varepsilon_{\mathrm{r}}^{8}+ \\
& 0.00000087 \varepsilon_{\mathrm{r}}^{9}
\end{aligned}
$$

where, $\mathrm{h}=$ Separation between the conductors, and $\varepsilon_{\mathrm{r}}=$ relative permittivity of the dielectrics.

\section{Coupled Line Structure}

When transmission lines are close together on a single plane, direct edge coupling between them is relatively high. But where one conductor is above the other, broadwall coupling occurs and the coupling is significantly higher than for edge coupled lines with the same separation..

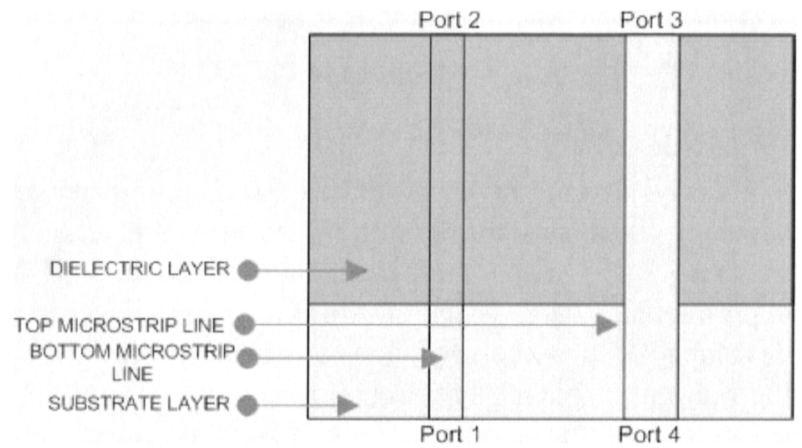

Fig. 4. Planar view of the coupled lines structure

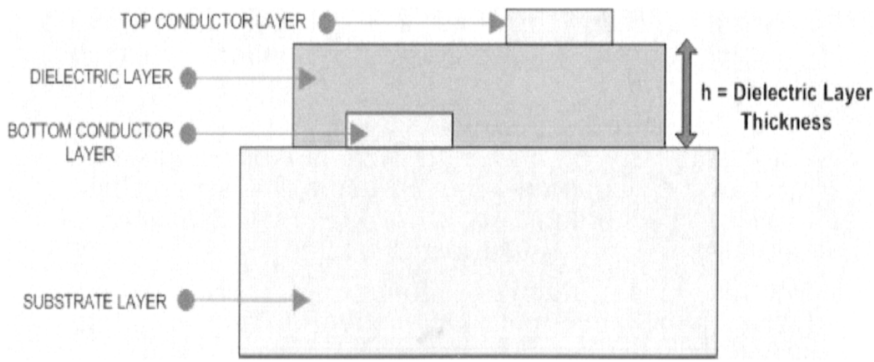

Fig. 5. Cross section of the coupled lines structure

With the aid of Momentum ${ }^{\circledR}$, the behaviour of the multilayer coupled line structure shown in Fig. 5. was analyzed at $100 \mathrm{GHz}$.

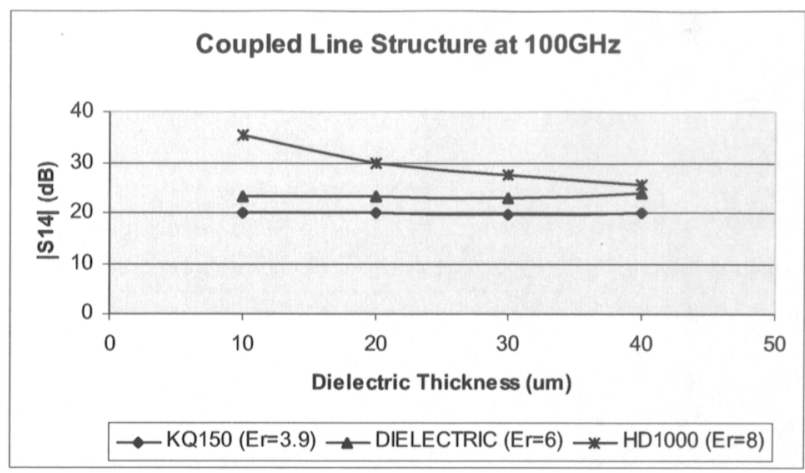

Fig. 6. Coupling of the coupled line structure

The coupling varies very slightly as the dielectric thickness between the conductors is increased, although the dielectric with a permittivity of 8 showed a significant decrease in coupling as the separation thickness was increased.

A curve fitting routine was used to determine relationships between the amount of coupling and the spacing, $h$, for different values of $\varepsilon_{\mathrm{r}}$ with the data obtained. Therefore it was concluded that for the conditions, $\mathrm{S} 11|>20 \mathrm{~dB},| \mathrm{S} 12|<0.5 \mathrm{~dB},| \mathrm{S} 13 \mid>10 \mathrm{~dB}$, $|\mathrm{S} 14|>10 \mathrm{~dB},|\mathrm{~S} 33|>25 \mathrm{~dB}$, and $|\mathrm{S} 43|<0.5 \mathrm{~dB}$, and for $3.9 \leq \varepsilon_{\mathrm{T}} \leq 8$, the minimum spacing required to achieve sufficient isolation between transmission lines, is given by:

$$
\mathrm{h}=46.857-3.42878 \varepsilon_{\mathrm{T}}+0.508 \varepsilon_{\mathrm{T}}^{2}-0.02 \varepsilon_{\mathrm{T}}^{3} .
$$

where, $\mathrm{h}=$ Separation between the conductors, and $\varepsilon_{\mathrm{T}}=$ relative permittivity of the dielectrics.

\section{Bend Structure}

In many cases where quite complex circuits are required on a single structure it is necessary to feed, between circuits using lengths of microstrip that include bends. These usually pass through an angle of $90^{\circ}$. Fig. 7 shows a typical bend configuration in a multilayer circuit.

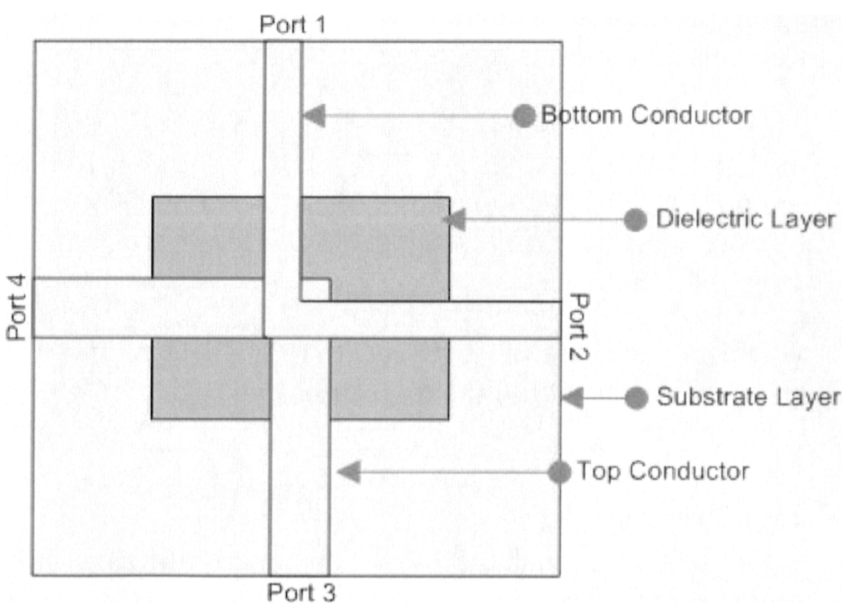


Fig.7. Planar view of the bend structure

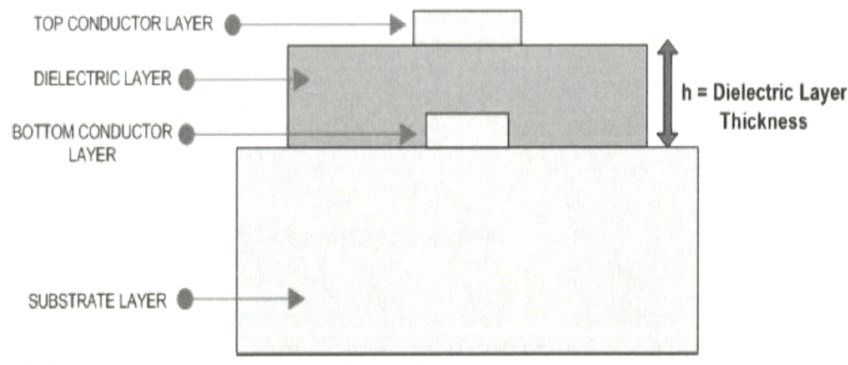

Fig. 9. Cross section of the bend structure

Results of the multilayer bend structure is shown in Fig. 10 at $100 \mathrm{GHz}$.

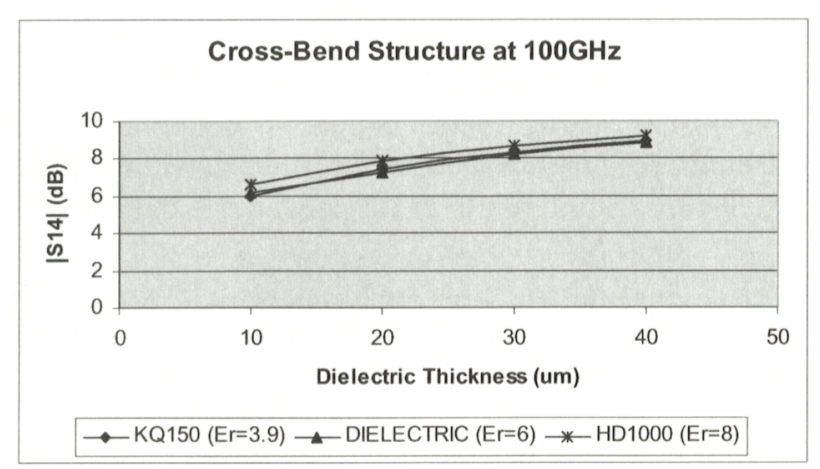

Fig. 10. Coupling of the bend structure

Over the range of dielectric thickness investigated, there appeared to be significant coupling between the right-angled conductors. The coupling was greater than that observed with the previous structures for the same dielectric thickness. This is a not unreasonable result, given that the right angled bend is a far more significant discontinuity. Following the technique used previously, a curve fitting method was used to determine a relationship between the amount of coupling and the spacing, $h$, for different values of $\varepsilon_{\mathrm{r}}$. Thus for the conditions, $|\mathrm{S} 11|>$ $10 \mathrm{~dB},|\mathrm{~S} 12|<2 \mathrm{~dB},|\mathrm{~S} 13|>10 \mathrm{~dB},|\mathrm{~S} 14|>7 \mathrm{~dB},|\mathrm{~S} 33|>$ $9 \mathrm{~dB}$, and $|\mathrm{S} 43|<2 \mathrm{~dB}$, and for $3.9 \leq \varepsilon_{\mathrm{r}} \leq 8$, the minimum spacing required to achieve sufficient isolation between transmission lines, is given by:

$\mathrm{h}=280.461-80.308 \varepsilon_{\mathrm{r}}-1.777 \varepsilon_{\mathrm{r}}^{2}+1.0723 \varepsilon_{\mathrm{r}}^{3}+0.1448 \varepsilon_{\mathrm{r}}^{4}$ $+0.00341 \varepsilon_{\mathrm{r}}^{5}-0.00172 \varepsilon_{\mathrm{r}}^{6}-0.00032 \varepsilon_{\mathrm{r}}^{7}-0.00002 \varepsilon_{\mathrm{r}}^{8}+$ $0.0000051 \varepsilon_{\mathrm{r}}^{9}$

where, $\mathrm{h}=$ Separation between the conductors, and $\varepsilon_{\mathrm{r}}=$ relative permittivity of the dielectrics.

\section{Java Program}

A software package was developed utilizing Java programming language for the circuit designer to obtain the minimum spacing between conductor in a multilayer package. It enables the design engineer to enter the desired circuit requirements, and executes the program. The minimum spacing is given in (um). Fig. 11 illustrates the screen dump of the executed program and Fig. 12 demonstrates the result calculated by the software along with the corresponding equation for minimum spacing between conductors in a multilayer circuit structure.

\begin{tabular}{|c|c|c|}
\hline \multicolumn{2}{|l|}{ STRUCTURES v1.0 } & -0 \\
\hline \multirow{4}{*}{$\begin{array}{l}\text { Structure type: } \\
\text { Cross-over } \\
\text { Couple Line } \\
\text { Bend }\end{array}$} & Frequency:- & 5-parameter:- \\
\hline & $20 \mathrm{GHz}$ & O $\$ 11$ \\
\hline & $60 \mathrm{GHZ}$ & $\mathbf{S 1 2}$ \\
\hline & $100 \mathrm{GHz}$ & S13 (NA: Cross-over) \\
\hline \multicolumn{2}{|l|}{ Dielectric permittivity:- } & $\begin{array}{l}\mathbf{S 1 4} \\
\mathbf{s 3 3}\end{array}$ \\
\hline $3.9 \bigcirc 5.0$ & 6.0 & $\mathbf{S 4 3}$ \\
\hline$\bigcirc 7.0 \bigcirc 8.0$ & & ALL \\
\hline Display Result & Reset & Exit \\
\hline
\end{tabular}

Fig. 11. Executable java program

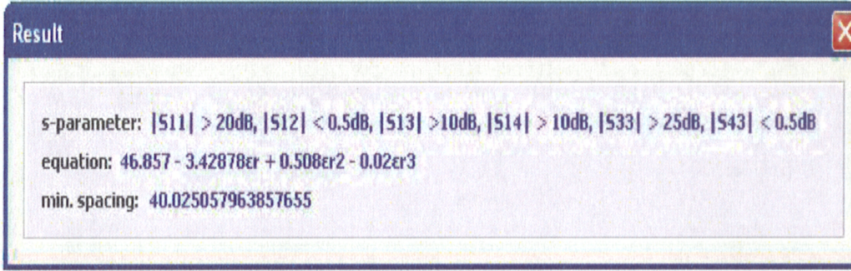

Fig. 12. Displaying of the results

\section{Conclusion}

Clearly, in multilayer structures the coupling between adjacent lines should be minimized to achieve signal transmission with minimal interference. Using experimental data, general design rules have been developed to provide guidance to the circuit designer on the minimum spacing between conductors in a multilayer package, and these rules have been incorporated into a simple software routine.

\section{References}

1. Mongia R., Bahl I. J., Bhartia P., RF and Microwave Coupled Line Circuits, Boston ; London : Artech House, c1999.

2. Tian Z. , Free C. E, Barnwell P.G, Woods J., Aitchison' C. S, "Design of Novel Multilayer Microwave Coupled-Line Structures Using Thick Film Technology", European Microwave Conference, pp. 1-4, October 2001.

3. Matthaei G. L., Young L., Jones E.M.T., Microwave filters, impedance-matching networks, and coupling structures, Dedham, Mass. : Artech House, 1980. 
